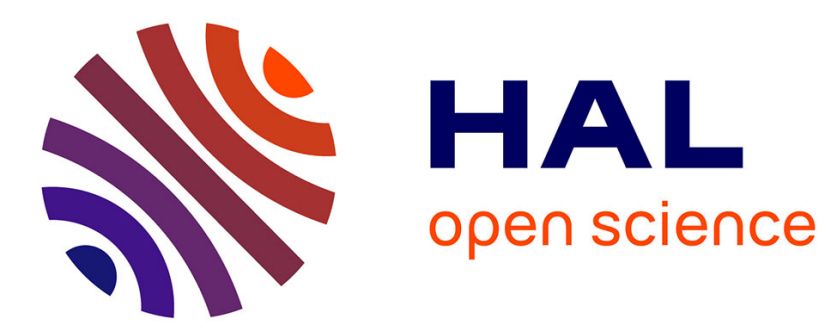

\title{
A Smart Grid WSN Research Testbed
}

Farah Abdel Khalek, Jad Nassar, Vincent Lefevere, Flovic Gosselin, Nicolas Gouvy

\section{To cite this version:}

Farah Abdel Khalek, Jad Nassar, Vincent Lefevere, Flovic Gosselin, Nicolas Gouvy. A Smart Grid WSN Research Testbed. WiMob 2020 - The 16th International Conference on Wireless and Mobile Computing, Networking and Communications., Oct 2020, Thessaloniki, Greece. hal-02991149

\section{HAL Id: hal-02991149 \\ https://hal.science/hal-02991149}

Submitted on 5 Nov 2020

HAL is a multi-disciplinary open access archive for the deposit and dissemination of scientific research documents, whether they are published or not. The documents may come from teaching and research institutions in France or abroad, or from public or private research centers.
L'archive ouverte pluridisciplinaire HAL, est destinée au dépôt et à la diffusion de documents scientifiques de niveau recherche, publiés ou non, émanant des établissements d'enseignement et de recherche français ou étrangers, des laboratoires publics ou privés. 


\title{
A Smart Grid WSN Research Testbed
}

\author{
Farah Abdel Khalek*, Jad Nassar*, Vincent Lefevere*, Flovic Gosselin ${ }^{\dagger}$ and Nicolas Gouvy ${ }^{\dagger}$ \\ * Computer Science and Mathematics Departement, Yncréa Hauts-de-France, Lille, France \\ \{farah.abdel-khalek, jad.nassar, vincent.lefevere\}@yncrea.fr \\ †Université Catholique de Lille, Lille, France \\ \{flovic.gosselin, nicolas.gouvy\}@univ-catholille.fr
}

\begin{abstract}
The increasing integration of renewable energy resources (e.g., photo-voltaic cells, wind farms) has turned traditional electric grids into smart ones. This evolution takes an active role in smart energy systems, especially when integrating a Wireless Sensor Network (WSN) to control and manage the grid. However, monitoring the whole grid with constrained devices is a challenge worth considering. In this paper, we introduce a WSN demonstrator for Smart Grids as part of the SoMel SoConnected project. It consists of Raspberry Pi and Zolertia ReMote sensor nodes deployed all over the building of the Catholic University of Lille and Yncréa Hauts de France. Thus, providing a WSN testbed in a real Smart Grid environment. A performance evaluation is conducted in the wired and wireless architectures in order to test some of the metrics that could be evaluated in this testbed, particularly the end to end delay and the packet delivery ratio.
\end{abstract}

Index Terms-Smart Grids, Wireless Sensor Networks, Contiki OS, Zolertia RE-Mote, Raspberry Pi, MQTT , Modbus, ZigBee

\section{INTRODUCTION}

The transformation of traditional power grids into "smarter" ones has become more and more common. This evolution, called the Smart Grids (SG), is manifested by (1) the integration of renewable energy resources all over the grid [1], (2) a two-way communication between the utility and the customers and (3) automated decisions of the smart connected devices. Therefore, these changes require the ability to exchange a maximum of data over the network, in order to monitor and control the different heterogeneous decentralized energy resources. A Wireless Sensor Network (WSN) distributed across the grid, on the different measuring and control points, is a potent and plausible solution [2]. However, sensor nodes are highly constrained devices. Their limitations in terms of power are due to their continuous radio transmission. Although WSN can drive a SG, it cannot always be powered by it due to the intermittent energy resources (i.e., renewable energy resources). Moreover, and on many levels in the power grid, such high voltage will make it challenging in terms of sensor placement and electric supply. For that, and in order to meet the Quality of Service (QoS) requirements of the different SG applications (e.g., delay tolerance, reliability, etc), a WSN controlling/monitoring a SG have to take these challenges into consideration [3].

Launched in 2013 by the Catholic University of Lille, LiveTREE represents the university's program for energy and social transition. It registers under Rev3 - the Third Industrial Revolution in the Hauts-de-France region through a collaborative approach between the students, personnel, residents, companies and collectivities. The project aims to reduce the campus' carbon footprint and transform it to a living laboratory of social innovation. The innovative solutions envisioned focus on the technical and human aspects of the transition and are tested in real conditions on the campus and its neighborhoods. The energy aspects are particularly explored in LiveTREE ${ }^{1}$, notably with the SoMEL SoConnected project which is part of YOU \& GRID ${ }^{2}$. The aim of this project is to enable the development of energy distribution networks to serve a territory through the connection of infrastructures. In light of that mission, the Faculties of the Catholic University of Lille and the Yncréa Hauts-de-France engineering school are developing self-consumption models of renewable energy in the non-residential sector. In this project, they test the technical feasibility and profitability of self-consumption models in the non-residential sector with building-integrated photo-voltaic, by piloting charges including recharging electric vehicles in private car parks. As part of this project, a wireless sensor demonstrator is developed. It consists of 120 Raspberry Pi and Zolertia sensor nodes distributed all over the building of the University.

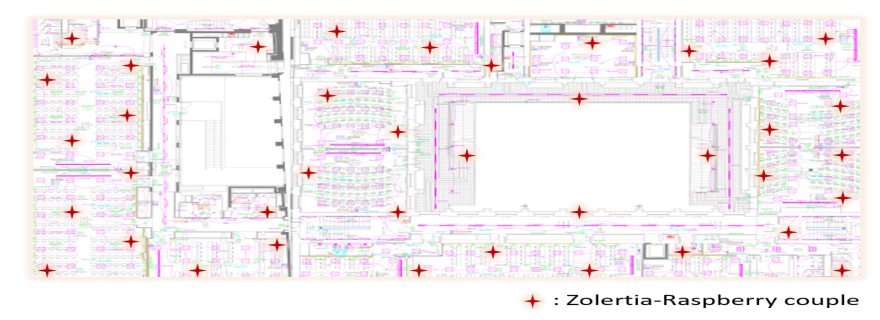

Fig. 1: Example of sensor deployment in one floor in the university

The aim of this demonstrator is to collect different sensor values from the photo-voltaic cells, electric vehicles, electric meters, etc. in order to control and pilot the energy production/consumption on campus. More precisely, two network architectures are implemented: the first one having a wired connection between the different measuring points and the sensors, based on Raspberry Pi version 3 (RPI) modules. The second one relies on low power wireless personal area (6LoWPAN) networks with Zolertia Re-Mote sensors. The

\footnotetext{
${ }^{1}$ http://livetree.fr/

${ }^{2}$ https://www.enedis.fr/yougrid-northern-france
} 
collected data are sent over both networks via a MQTT broker/client communication and are stored in a database for a future use. It is important to mention that the wired network is used to control, monitor and reprogram the wireless sensor network (e.g., detect transmission loss, provide a benchmark for wireless routing measurements, etc.).

We note that the physical installation of the sensors in the building is in process. Figure 1 shows an example of the sensor deployment on one floor of one of the buildings. We used the range-test application [4] in order to ensure an efficient and adequate placement of the Zolertia Re-Mote nodes.

The remainder of this paper is organized as follows. Section II presents a summary of the previous work on WSN demonstrators. Section III-A and III-B introduce the hardware and software tools used for the design and deployment of the demonstrator's WSN. Section IV illustrates a performance comparison between a WSN and a wired network in a realcase environment. Finally, section V concludes the paper.

\section{STATE OF THE ART}

The heterogeneity of IoT applications leads to a large diversity of WSN testbeds, supporting different topologies and network layer protocol options. Many wireless sensor network testbeds are available to the community today. In [5], the authors present FIT IoT-LAB, an open testbed that consists of 2728 motes located in 6 different sites across France, offering an accurate open access and open source multi-user scientific tool. It provides repeatable mobility via electric toy trains, multi-site experiments and energy consumption measurement. In [6], TWIST supports experiments with diverse node platforms, active power supply and creation of hierarchical and flat sensor networks control of the nodes. In [7], SmartSantander offers advanced hardware, scale, functionalities proposed to the user, mobility via public buses Multi-site and real-life experiments. In [8] and [9], other WSN testbeds were developed. However, all of these demonstrators generally operate in controlled environments and are not so open to public.

\section{Global Architecture}

Our demonstrator aims to provide a WSN testbed in a real SG environement. Collected data are provided by PV cells, electric vehicles, electrical equipment, etc. The communication is ensured via 120 sensor nodes deployed all over the buildings of the university. In the following subsections, we will briefly describe the network's hardware and software architectures.

\section{A. Hardware Architecture}

The testbed consists of RPI-Zolertia sensor nodes, communicating over two types of networks (wired and wireless). In figure 2, the global architecture of our testbed and both wired and wireless networks are presented. The wired network serves as a controller to reprogram the wireless nodes, collect data from the different electrical equipment and store it in the database on the server. In a detailed view, a sensor node in our architecture consists of a RPI-Zolertia couple. It is a master-slave type of relation, where RPIs control and manage the wireless network, while Zolertia devices assume the role of processing and routing the received information from the different energy sources.

The wireless network is composed of three types of nodes: (1) source, (2) relay and (3) destination nodes. Source nodes are responsible of reading data from the RPIs, processing and transmitting it to a one hop relay node. Relay nodes receive the data from the source nodes and then take care of path computing, processing, and communicating to destination nodes across the wireless network. Finally, destination nodes process the received data packets to execute specific tasks (i.e., storing them in the database via the RPI). It is important to note that the Zolertia family is composed of different generations (e.g., Z1, Re-Mote, Firefly). In our demonstrator, we used Zolertia Re-Mote as a fast development tool for testing our IoT application. However, in the final network architecture, Firefly nodes [10], a more advanced generation, were the ones implemented.

\section{B. Software Architecture}

As already mentioned, our sensor node consists of a RPIZolertia couple. Firstly, RPI nodes are programmed using Python language. They serve first of all to reprogram/reflash the Zolertia nodes. Moreover, they communicate with the PV cells and the electrical equipment using the Modbus TCP/IP Protocol [11]. It is used to establish a master-slave Ethernet communication between different devices. Each slave in the network is assigned a unique unit address and stores data as a numerical format in tables called registers. The different Modbus equipment are all connected to a measurement center (D-50 AGBT) via a Digiware Bus. Once the RPI node connects to that center, it chooses the corresponding slaves to address, as well as the desired registers to read from. In other words, the RPI node will choose the data type that it needs to get from the electrical equipment in order to transmit it afterwards to the Zolertia node. Its worth mentioning that we used ModbusDoctor, a real-time simulator. Next, and after the data collection from the electric equipment on the RPI level, these data are transmitted to the Zolertia nodes via a serial communication. These nodes are programmed to receive and transmit data in radio signals, taking into account their limited energy resources. Contiki [12], a largely used operating system for memory-constrained devices, is implemented on these sensor nodes, thus enabling the connection to IPv4/IPv6. For that, devices are configured to meet the aforementioned standards and to reach every node in the network. This is done by implementing a 6LoWPAN [13] network. This protocol delegates all the capabilities of IPv6 on a constrained device. On the application layer, and in order to distribute the collected data more efficiently, Message Queue telemetry transport (MQTT) [14], a lightweight publish and subscribe protocol, is implemented on both the Zolertia and RPI nodes. A local broker running on the server side is responsible for handling the publishing and subscribing events. On the other side, each sensor node (wired and wireless) publishes the 


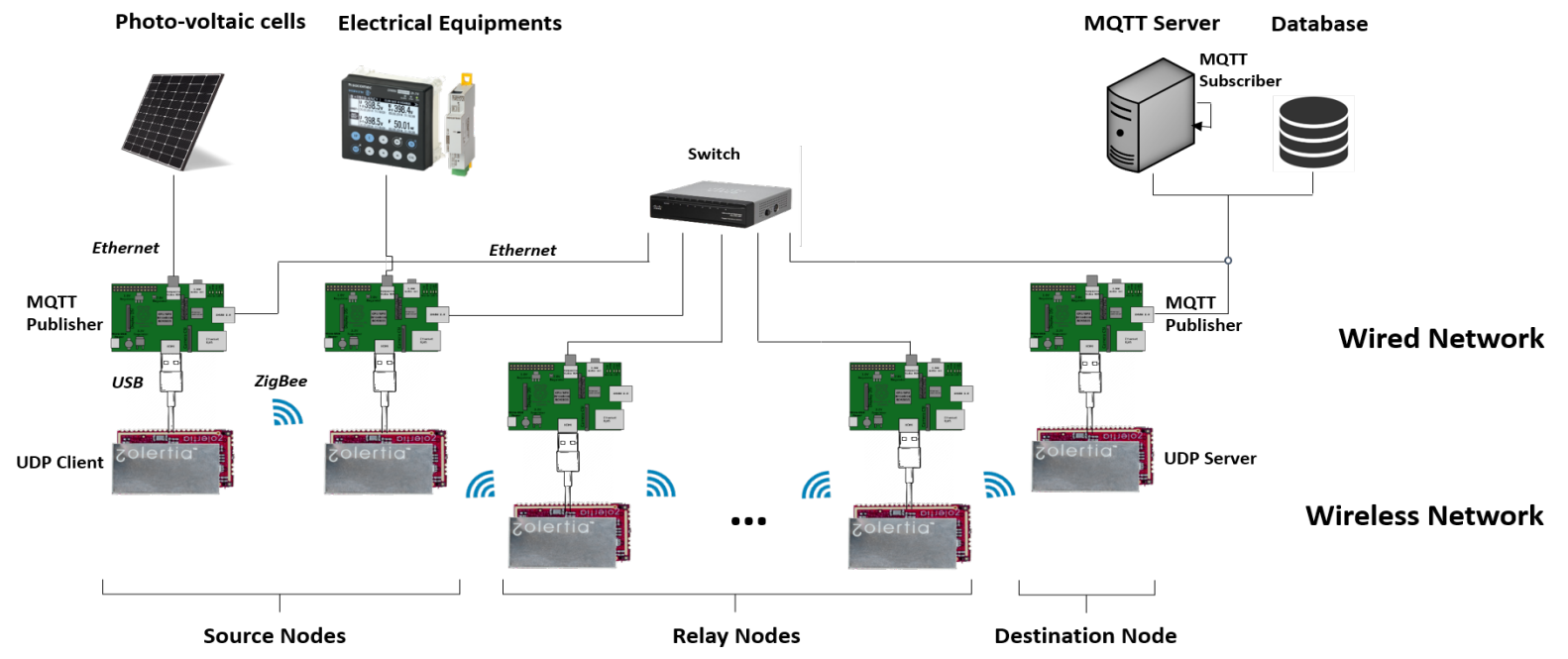

Fig. 2: Global Architecture Overview

captured values in distinct topics. Data is then handled and inserted in the database by the subscriber.

\section{EXAMPLE OF USE CASES}

In order to test our network, a small use case scenario composed of three RPI-Zolertia sensor nodes (source, relay and destination) is studied. These nodes were deployed in a typical office environment in the university's building.

It it important to note that the current use case is just an example to show some of the metrics that can be evaluated/measured in our testbed. For that the comparison between the wireless and the wired networks is not intended to evaluate or to compare the performance of one or the other.

The network architecture implemented is similar to the one depicted in figure 2. On one hand, a reliable, non-intrusive and real-time system is implemented using RPI modules. They are connected all together by an SLM2008 Cisco switch, thus establishing a wired network. Thereafter, each module is given access to the database by authenticating to a web service. It then connects to the Modbus measurement center, and fetch the PV and electrical equipment's real-time values. Once fetched, these values are inserted via MQTT in the database. On the other hand, a wireless low-power network is also implemented, using the Zolertia Re-Mote sensors, where each one is connected to a RPI module. In this case, three scenarios take place: the first concerns the Zolertia source node connected to the RPI source. It retrieves the previously collected values (by the RPI from the PV cells and electrical equipment) and initiates the propagation process to the rest of the nodes by sending the data packets over the network. The second scenario illustrates the relay node, which mission consists of routing the packets from source to destination via the wireless medium. On the receiving edge, the destination node transmits the packet to the connected RPI, through serial cable, to be inserted in the database for a future use.

\section{A. Experiment Parameters}

In order to evaluate our approach, the experiment was performed on Contiki OS using the RPI-Zolertia sensor nodes as already mentioned. Experimentation parameters are presented in Table I. Tests were repeated 3 times. The topology consists of 3 sensor nodes placed at a distance of approximately 15 meters from each other. They are configured to transmit their radio signals on channel 26 , on the $2.4 \mathrm{GHz}$ frequency band. The client sends the collected measurements as UDP packets [15] every 5 seconds using the RPL routing protocol [16] on the network layer. A UDP server running on the destination node, sets up the UDP connection and waits for packets from clients.

\begin{tabular}{|ll|}
\hline Parameters & Values \\
\hline Sensors & Zolertia Re-Mote \\
\hline Operating System & Contiki \\
\hline Comm. Protocols & RPL, UDP, MQTT \\
\hline Channel & 26 \\
\hline Frequency & $2.4 \mathrm{GHz}$ \\
\hline Distance between nodes & Approximately 15 meters \\
\hline Number of nodes & 1 client, 1 relay and 1 server \\
\hline Sending interval & 1 packet every 5 seconds \\
\hline
\end{tabular}

TABLE I: Parameters of the experimentation

\section{B. Performance Evaluation}

Figure 3 shows the percentage of packet loss during a period of 75 minutes. We notice that, in the wireless network, it is a non-stable curve, given the distinct signals that may interfere in the experiment's environment (e.g., WiFi). As expected, the wired network does not present any packet loss.

Figure 4 shows the resulted delay between the sent and received packets from the source to the destination node, in both the wired and wireless networks. We observe a delay ranging from 1.2 to 1.7 seconds in the wireless network. However, no delay (negligible) is recorded in the wired one. 


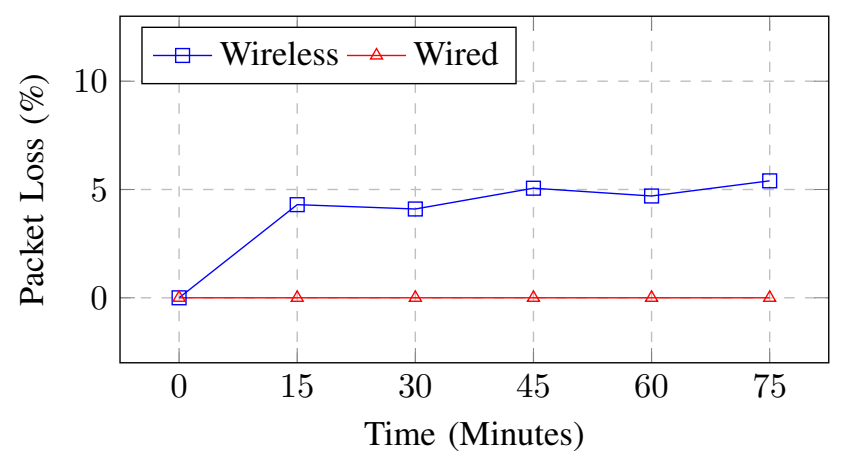

Fig. 3: Packet loss percentage for wireless and wired networks

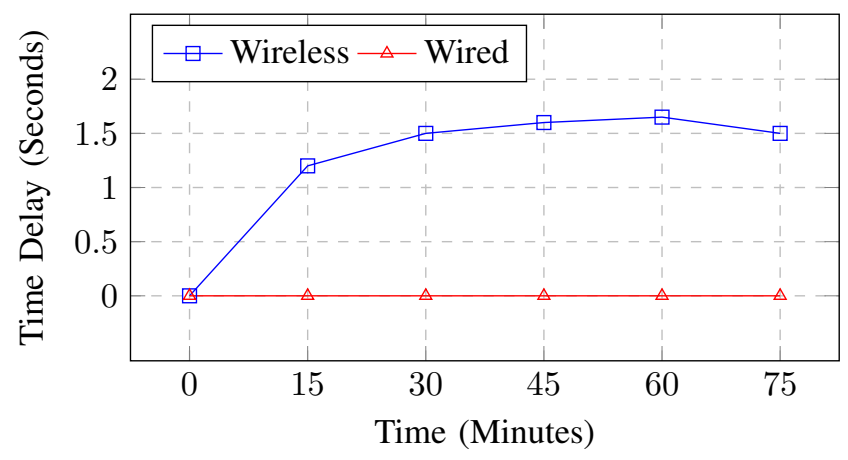

Fig. 4: Time delay for wireless and wired networks

\section{Conclusion, Actual State and Future Work}

In this paper, we introduced a SG WSN testbed that was developed in the SoMEL SoConnected project. It consists of a set of 120 RPI-Zolertia sensor nodes that are being deployed all over the building of the Catholic University of Lille and Yncréa Hauts de France. The purpose of developing this demonstrator is to monitor and control the energy production and consumption on campus via a WSN. This testbed is still under-deployment, and will be open to public access once fully implemented. However, a live experiment was done using 3 sensor nodes (source, relay and destination), in order to evaluate our testbed. The results show a packet loss percentage (varying between $4 \%$ and $5 \%$ starting the minute 15 of the experiment) in the wireless network. Unlike the wired one where no packet loss percentage is noticeable. Another metric was studied in order to observe the time delay from the source to the destination. Results show a delay of approximately 1.5 seconds in the wireless network, and a negligible delay in the wired one.

In the near future, we will continue the physical deployment of the sensor nodes and the development of a dedicated website open to public in order to be able to launch experiments in a user friendly environment. This access will be done while ensuring the security of our equipment (i.e., developing the corresponding API to control the access). This network of sensors will make it possible to experiment, in real conditions (radio interference, crossing thick walls, loss of signal, etc.), specific self-adaptive communication protocols necessary for managing a Smart Grid such as the differentiated routing of control orders and/or production or electricity consumption measurements.

\section{ACKNOWLEDGMENTS}

The project SoMel SoConnected was funded by a grant from the Ademe (PIA2). This project involves the MEL (Métropole Européenne de Lille), Enedis, EDF, Dalkia, Intent, the Lille Economie-Management Laboratory, HEI - Yncréa HdF and the Faculties of the Catholic University of Lille.

\section{REFERENCES}

[1] N. Phuangpornpitak and S. Tia, "Opportunities and challenges of integrating renewable energy in smart grid system," Energy Procedia, vol. 34, pp. 282-290, 2013.

[2] S. Rekik, N. Baccour, M. Jmaiel, and K. Drira, "Wireless sensor network based smart grid communications: Challenges, protocol optimizations, and validation platforms," Wireless Personal Communications, vol. 95, no. 4, pp. 4025-4047, 2017.

[3] J. Nassar, M. Berthomé, J. Dubrulle, N. Gouvy, N. Mitton, and B. Quoitin, "Multiple instances qos routing in rpl: Application to smart grids," Sensors, vol. 18, no. 8, p. 2472, 2018.

[4] A. Lignan, "Range tests made easy with the re-mote and lcd," Tech. Rep., 2016. [Online]. Available: https://www.hackster.io/alinan/rangetests-made-easy-with-the-re-mote-and-lcd-6e78b3

[5] C. Adjih, E. Baccelli, E. Fleury, G. Harter, N. Mitton, T. Noel, R. Pissard-Gibollet, F. Saint-Marcel, G. Schreiner, J. Vandaele et al. "Fit iot-lab: A large scale open experimental iot testbed," in 2015 IEEE 2nd World Forum on Internet of Things (WF-IoT). IEEE, 2015, pp. 459-464

[6] V. Handziski, A. Köpke, A. Willig, and A. Wolisz, "Twist: a scalable and reconfigurable testbed for wireless indoor experiments with sensor networks," in Proceedings of the 2nd international workshop on Multihop ad hoc networks: from theory to reality, 2006, pp. 63-70.

[7] M. Nati, A. Gluhak, H. Abangar, and W. Headley, "Smartcampus: A user-centric testbed for internet of things experimentation," in 2013 16th International Symposium on Wireless Personal Multimedia Communications (WPMC). IEEE, 2013, pp. 1-6.

[8] J. Albesa, R. Casas, M. T. Penella, and M. Gasulla, "Realnet: An environmental wsn testbed," in 2007 International Conference on Sensor Technologies and Applications (SENSORCOMM 2007). IEEE, 2007, pp. 502-507.

[9] J. Schaerer, Z. Zhao, J. Carrera, S. Zumbrunn, and T. Braun, "Sdnwisebed: A software-defined wsn testbed," in International Conference on Ad-Hoc Networks and Wireless. Springer, 2019, pp. 317-329.

[10] N. J. Mouri, "Iot protocols and security," Information Technology, 2019.

[11] A. Swales et al., "Open modbus/tcp specification," Schneider Electric, vol. 29,1999

[12] A. Dunkels, B. Gronvall, and T. Voigt, "Contiki-a lightweight and flexible operating system for tiny networked sensors," in 29th annual IEEE international conference on local computer networks. IEEE, 2004, pp. 455-462.

[13] N. H. Kumar, S. Baskaran, S. Hariraj, and V. Krishnan, "An autonomous aquaponics system using 6lowpan based wsn," in 2016 IEEE 4th International Conference on Future Internet of Things and Cloud Workshops (FiCloudW). IEEE, 2016, pp. 125-132.

[14] U. Hunkeler, H. L. Truong, and A. Stanford-Clark, "Mqtt-s-a publish/subscribe protocol for wireless sensor networks," in 2008 3rd International Conference on Communication Systems Software and Middleware and Workshops (COMSWARE'08). IEEE, 2008, pp. 791798.

[15] G. Oikonomou and I. Phillips, "Experiences from porting the contiki operating system to a popular hardware platform," in 2011 International Conference on Distributed Computing in Sensor Systems and Workshops (DCOSS). IEEE, 2011, pp. 1-6.

[16] T. Clausen, U. Herberg, and M. Philipp, "A critical evaluation of the ipv6 routing protocol for low power and lossy networks (rpl)," in 2011 IEEE 7th International Conference on Wireless and Mobile Computing, Networking and Communications (WiMob). IEEE, 2011, pp. 365-372. 